INPLASY

PROTOCOL

To cite: Wan et al. Effect of brain computer interface rehabilitation training on functional rehabilitation of stroke patients: A protocol for meta-analysis. Inplasy protocol 202170067. doi:

10.37766/inplasy2021.7.0067

Received: 21 July 2021

Published: 21 July 2021

Corresponding author:

Li Yongqiang

liyongqiang_1980@163.com

Author Affiliation:

The Center of Rehabilitation Medicine, The First Affiliated Hospital of Nanjing Medical University, Nanjing, Jiangsu Province, China.

Support: None.

Review Stage at time of this submission: Formal screening of search results against eligibility criteria.

Conflicts of interest:

None declared.

\section{Effect of brain computer interface rehabilitation training on functional rehabilitation of stroke patients: A protocol for meta-analysis}

\author{
Wan, C1; Qiu, H2; Wang, X3; Ge, P4; Huang, S5; Wang, Z6; Li, Y7.
}

Review question / Objective: Systematically evaluate the effect of brain-computer interface $(\mathrm{BCl})$ training on the rehabilitation of stroke patients.

Condition being studied: At present, stroke is one of the main causes of disability in adults, with a disability rate as high as $60 \%-80 \%$, bringing a heavy economic burden to the country and many families. Therefore, more effective stroke rehabilitation strategies are needed. With the development of rehabilitation medicine technology and artificial intelligence technology, brain-computer interface $(\mathrm{BCI})$ and other technologies that directly intervene the central nervous system have been continuously studied and applied in clinical treatment to help patients recover their mobility and support motor function recovery after stroke. Numerous RCTs have investigated the effects of $\mathrm{BCl}$ training on motor and brain function recovery in post-stroke patients, and although these studies have shown significant effects on recovery, the pitfalls of the studies are the small number of participants, the small number of training sessions, and/or the lack of follow-up assessments. The overall goal of this study is to summarize the evidence from RCTs comparing brain-computer interfaces with other treatments in post-stroke patients, focusing on motor function.

INPLASY registration number: This protocol was registered with the International Platform of Registered Systematic Review and Meta-Analysis Protocols (INPLASY) on 21 July 2021 and was last updated on 21 July 2021 (registration number INPLASY202170067).

\section{INTRODUCTION}

Review question / Objective: Systematically evaluate the effect of brain-computer interface $(\mathrm{BCl})$ training on the rehabilitation of stroke patients.
Condition being studied: At present, stroke is one of the main causes of disability in adults, with a disability rate as high as $60 \%$ $-80 \%$, bringing a heavy economic burden to the country and many families. Therefore, 
more effective stroke rehabilitation strategies are needed. With the development of rehabilitation medicine technology and artificial intelligence technology, brain-computer interface (BCl) and other technologies that directly intervene the central nervous system have been continuously studied and applied in clinical treatment to help patients recover their mobility and support motor function recovery after stroke. Numerous RCTs have investigated the effects of $\mathrm{BCl}$ training on motor and brain function recovery in poststroke patients, and although these studies have shown significant effects on recovery, the pitfalls of the studies are the small number of participants, the small number of training sessions, and/or the lack of follow-up assessments. The overall goal of this study is to summarize the evidence from RCTs comparing brain-computer interfaces with other treatments in poststroke patients, focusing on motor function.

\section{METHODS}

Search strategy: The databases of PubMed, Web of science, The Cochrane Library, Embase, China National Knowledge Infrastructure (CNKI), Wan fang Data Knowledge Service Platform, Wan fang Medical Network, Chinese Biomedical Literature Service System (Sino Med), and VIP were searched by computer from database establishment to November 2020. In addition, the references of included articles were traced to supplement the acquisition of relevant articles. The retrieval adopts the mode of combining subject headings and free words. Chinese search terms included: brain-computer interface, human-computer interface, stroke rehabilitation, hemiplegic rehabilitation, cerebral infarction, cerebral hemorrhage. English search terms included: brain-computer interface, brainmachine interface, stroke, cerebrovascular accident, cerebral hemorrhage, cerebralin farction, rehabilitation.

Participant or population: Stroke patients; Race, nationality and duration of disease are not limited.
Intervention: Brain computer interface rehabilitation training.There are no restrictions on external control devices, training intensity, rating, upper or lower limbs for BCl.

Comparator: Control group: Non brain computer interface therapy, conventional physical therapy, or other therapeutic modalities.

Study designs to be included: Randomized controlled trials (RCTs).

Eligibility criteria: Inclusion criteria: (1)

Study type: Randomized clinical controlled trial on the effect of $\mathrm{BCl}$ training on functional rehabilitation in stroke patients;

(2) subjects: The study subjects were patients diagnosed with stroke, regardless of race, nationality, and course of disease;

(3) Outcome indicators: The main outcome indicator is Fugl-Meyer motor function score (upper limb FMA-UE or lower limb FMA-LE). Secondary indicators are the total score of Barthel index or modified Barthel index (BI or MBI) and modified Ashworth grade (MAS).Exclusion criteria: (1) The study is designed as selfcrossover controlled trial, uncontrolled clinical trial and other non-RCTs;(2) No definite clinical diagnosis; (3) Incomplete information, incorrect data or failure to extract relevant calculation indicators; (4)Repeated published literatures or periodical report of a study;(5) Review, comment or meeting minutes;(6) Articles with incomplete data and unavailable to contact authors

Information sources: We conducted a Systematic literature search in PubMed, Web of science, The Cochrane Library, Embase, CNKI, Wan Fang Data, Sino Med, VIP databases.

Main outcome(s): The main outcome indicator is Fugl-Meyer motor function score (upper limb FMA-UE or lower limb FMA-LE). 
Additional outcome(s): Secondary indicators are the total score of Barthel index or modified Barthel index (BI or MBI) and modified Ashworth grade (MAS).

Data management: Import the retrieved documents into EndnoteX9. The following information was extracted from all qualified studies by two researchers independently: (1)Basic information of included studies: study title, first author, publication journal, etc.;(2) Baseline characteristics and diagnostic information of study subjects;(3) Key elements of risk of bias evaluation;(4) Outcome measures and outcome measures of interest. Use Review Manager 5.1 software to assess the risk bias of all included qualified studies and perform data analysis.

Quality assessment / Risk of bias analysis: Two assessors evaluated the risk of bias of included RCTs using the tools recommended by the Cochrane Handbook 5.1.0, respectively, including: (1) Random sequence generation; (2) Allocation concealment; (3) Blinding of participants and personnel; (4) Incomplete outcome assessment; (5) Selective reporting; (6) other bias. Risk of bias was assessed according to the following criteria: "yes" indicates low risk of bias; "no" indicates high risk of bias, and "unclear" indicates uncertain risk of bias. Methodological quality evaluation was performed independently by two reviewers, and in case of disagreement, it was resolved by discussion.

Strategy of data synthesis: Meta-analysis was performed using RevMan $\mathbf{5 . 1}$ software. The pooled effect size was weighted mean difference (WMD) or standardized mean difference (SMD) and its $95 \% \mathrm{Cl}$. The heterogeneity test was performed first, and the $\mathrm{X} 2$ test was used to analyze the heterogeneity between the study results, combined with 12 judgment, with a test level of $a=0.1$. If the study results are homogeneous $(P>0.1,12<$ $50 \%$ ), the fixed-effect model is used for meta-analysis; if the study results are statistically heterogeneous $(P \leq 0.1,12 \geq$ $50 \%$ ), the causes of heterogeneity are analyzed. If there is clinical heterogeneity, sensitivity analysis or meta-analysis can be performed according to its source; if there is no significant clinical heterogeneity, the random-effect model is used for metaanalysis. Z-test was used for the pooled effect value, and the test level was $a=0.05$.

Subgroup analysis: When there is heterogeneity between studies, the method of subgroup analysis is often used to deal with it. Each variable such as study design, sample characteristics, length of treatment and so on can be divided into subgroups for analysis, If the results of subgroup analysis - indicate that each subgroup The group does not show heterogeneity, which suggests that this variable may be one of the sources of heterogeneity, which can reduce the heterogeneity caused by the difference of this variable.

Sensitivity analysis: Using Review Manager 5.1 software to assess the risk bias of the included all qualified studies. The size of heterogeneity of the studies was assessed based on the PP value: I $2 \geq 50 \%$ or $P<$ 0.05 indicated high heterogeneity. Sensitivity analysis is used to find the reasons for the heterogeneity, the random-effects model is used for metaanalysis.

Country(ies) involved: China.

Keywords: Stroke; Brain-computer interface; Rehabilitation; Function; Metaanalysis.

Contributions of each author:

Author 1 - Wan Chunli - Study design, guidance and drafted the manuscript.

Email: wanchunliw@163.com

Author 2 - Qiu Huaide - Involved in the design of the study.

Author 3 - Wang Xue - Literature search and selection.

Email: wangxue312@yahoo.net

Author 4 - Ge Panli - Literature search and selection.

Email: 790340350@qq.com 
Author 5 - Huang Sisi - Data collection and synthesis.

Email: hss0904@163.com

Author 6 - Wang Zhixiang - Data collection and synthesis.

Email: wangzhixiang.hao@163.com

Author 7 - Li Yongqiang - Study design, guidance.

Email: liyongqiang_1980@163.com 